\title{
Effects of step salinity gradients on salmon lice larvae behaviour and dispersal
}

\author{
T. Crosbie ${ }^{1}$, D. W. Wright ${ }^{2}$, F. Oppedal ${ }^{2}$, I. A. Johnsen ${ }^{2}$, F. Samsing ${ }^{1,3}$, T. Dempster ${ }^{1, *}$ \\ ${ }^{1}$ Sustainable Aquaculture Laboratory_Tropical and Temperate (SALTT), School of BioSciences, University of Melbourne, \\ Victoria 3010, Australia \\ ${ }^{2}$ Matre Aquaculture Research Station, Institute of Marine Research, 5984 Matredal, Norway \\ ${ }^{3}$ CSIRO, Castray Esplanade, Battery Point, Tasmania 7004, Australia
}

\begin{abstract}
In trying to deal with the problematic salmon louse Lepeophtheirus salmonis in salmon aquaculture, strategies to better prevent infestations are gaining traction. Successful prevention requires an accurate understanding of the environmental influences that alter the distribution of the planktonic stages of lice in the water column in space and time. Here, we tested the salinity preferences of nauplii and copepodid larval stages using step salinity column experiments. Under consistent temperature and lighting conditions, we created step gradients using a bottom layer of full salinity (34.7 ppt), with an upper layer of equal or lower salinity ( 34.7 to $16 \mathrm{ppt}$ ). Lice entered the column in the lower layer and dispersed for $1 \mathrm{~h}$ before their position was recorded. Both nauplii and copepodids increasingly avoided the overlying layers as they became more brackish. However, the strength of avoidance differed between nauplii and copepodids. Nauplii almost completely avoided salinities below 30 ppt. For copepodids, there was a more gradual decline in the proportion preferring the less saline overlying layer, and the presence of some individuals occurred even at 16 to 20 ppt. Both stages aggregated at or just below the halocline, with no aggregation evident in isohaline columns at the same depth. For nauplii, clustering within the halocline was particularly strong. When integrated into a sea lice dispersal model, the new salinity preferences we determined markedly altered dispersal patterns in scenarios when salinity gradients were present. Our results have implications for the mapping of salmon lice larval behaviour and dispersal, with benefits for aquaculture planning and management.
\end{abstract}

KEY WORDS: Halocline $\cdot$ Lepeophtheirus salmonis $\cdot$ Model $\cdot$ Sea lice $\cdot$ Vertical distribution

\section{INTRODUCTION}

The salmon louse Lepeophtheirus salmonis is a common marine ectoparasite that has become a major problem for both wild and farmed salmonids. In their parasitic phase, lice feed on the mucus, epidermis and blood of infected fish, and severe infestations can result in death (Torrissen et al. 2013). Infected Atlantic salmon Salmo salar have altered osmoregulation (Tully \& Nolan 2002, Hamre et al. 2013) and metabolic functions (Dawson et al. 1999), and can develop lesions, leaving them vulnerable to

*Corresponding author: dempster@unimelb.edu.au secondary infections. Furthermore, high-density infection can reduce swimming ability and oxygen intake, compromising swimming performance (Wagner \& McKinley 2004, Bui et al. 2016). Within salmon farms, high lice densities reduce productivity and fish welfare (Øverli et al. 2014).

The proliferation of lice in intensive farming regions has also led to increased parasitic loads on wild salmonids, with negative effects on wild populations (Bjørn et al. 2001, Kristoffersen et al. 2018). To limit the size of spillback onto wild populations in areas of intensive aquaculture, regulations stipulate

(C) The authors 2019. Open Access under Creative Commons by Attribution Licence. Use, distribution and reproduction are unrestricted. Authors and original publication must be credited. 
levels which trigger anti-lice treatments (e.g. threshold of 0.5 adult females per fish in Norway; Lovdata 2018). Chemotherapeutic and mechanical treatments to remove lice are costly (Abolofia et al. 2017) and can cause fish mortality and reduce growth rates (Overton et al. 2018a,b).

As an alternative to treatments, preventative measures that aim to inhibit initial infection are rapidly being deployed. Many preventative measures are 'depth-based' and work on the principle that the infective copepodid planktonic stage predominantly occurs in surface waters. The use of deep lights and feeding (Frenzl et al. 2014), snorkels (Wright et al. 2017) and cage skirts (Grøntvedt et al. 2018, Stien et al. 2018) at the cage level all function on the depthbased principle by either holding fish deeper in the water column where lice abundances are lower (e.g. deep lights and feeding) or providing a protective barrier to prohibit entry of lice into the surface waters of cages (e.g. skirts and snorkels). The efficacy of these preventative measures varies in space and time, depending on the environmental conditions that modify the abundance and distribution of the infective salmon lice.

Preventing infection for both farmed and wild fish is also possible on a larger scale through being selective in where farms are placed and the extent to which they are stocked. Coupled hydrodynamicbiological models are being used throughout the world for management and ecological studies to understand the effect of sea lice on wild fish (Kristoffersen et al. 2013, Myksvoll et al. 2018). For example, a lice dispersal model forms the basis for Norway's new 'traffic light system' to plan production levels by zone (Vollset et al. 2018). The model in operation across Norway is the National Sea Lice Dispersal Model (NSLDM), which uses both oceanographic (currents, temperature and salinity) and biological factors (temperature-dependent development rates, swimming behaviour) to predict where lice will travel and how far (Myksvoll et al. 2018). Johnsen et al. (2014) determined that the horizontal distribution of lice from a source is largely reliant on the vertical distribution of the planktonic nauplii and copepodid stages of lice, which depends on environmental cues.

The non-parasitic nauplii and parasitic copepodid life history stages may differ in their responses to environmental cues. Nauplii must survive and develop to become copepodids, pursuing favourable and/or avoiding unfavourable conditions to do so (á Norði et al. 2015, Samsing et al. 2016b, Wright et al. 2016). Copepodids are infective and must find and attach to a host before depleting their energy reserves (Tucker et al. 2000). Their environmental preferences therefore likely produce a vertical distribution strategy that creates the greatest chance of encountering a suitable host. Like other plankton, nauplii and copepodids disperse long distances from their hatching locations via currents (e.g. Kinlan et al. 2005, Treml et al. 2008). Lice larvae can swim upwards at $0.5-1.55 \mathrm{~mm} \mathrm{~s}^{-1}$ and sink at a slower rate (Allen \& Lewis 2013). As the strength and direction of oceanographic factors often change with depth, dispersal models such as the Norwegian NSLDM (Johnsen et al. 2014, Myksvoll et al. 2018) require the best estimates of larval distribution with depth.

Multiple environmental stimuli impact larval position with depth. Salmon lice larvae are phototactic and migrate upwards towards light if other influences are not present (Heuch et al. 1995, Aarseth \& Schram 1999). Salinity also alters their vertical distribution; when given a choice between 2 step gradients, copepodids were attracted to highest salinity ( 30.4 ppt; Heuch 1995). Previous tests of the response of lice to salinity were only made for copepodids at 3 widely divergent step gradients (15.4, 25.4 and $28.4 \%$; Heuch 1995). As a consequence, the NSLDM sets 20 ppt as a sharp cut off for when copepodids begin to actively avoid low salinities in the water column. Given the importance of understanding the vertical distribution of lice to optimise preventative measures, finer-scale testing of responses towards salinity changes is required across the full range of salinity step gradients that may exist in fjord and coastal environments. Furthermore, given the different life-history stage strategies between nauplii and copepodids, their responses to salinity need to be explored separately. Johnsen et al. (2014) determined that the horizontal distribution of lice from a source is largely reliant on the vertical distribution of the planktonic nauplii and copepodid stages of lice, which depends on environmental cues.

Here, we aimed to determine the distribution of nauplii and copepodid stages in step salinity gradients and to use the data in refining modelling of lice larvae dispersal. We hypothesised that: (1) larvae would prefer layers with higher salinity, and (2) vertical distribution would vary with life history stage. We designed a column with a halocline of ocean-like salinity (34.7 ppt) on the bottom, with a range of salinities (16-34.7 ppt) in the overlying layer. Thereafter, we compared dispersal modelling outputs with pre-existing parameters and the newly determined parameters for a fjord-coastal area with intensive salmon farming. 


\section{MATERIALS AND METHODS}

\subsection{Production of nauplii and copepodids}

We first created a source population of salmon lice from which to produce nauplii and copepodids for subsequent salinity experiments. Adult female salmon lice Lepeophtheirus salmonis were sourced from Atlantic salmon held at a commercial farm in Western Norway $\left(60^{\circ} \mathrm{N}\right)$. Lice were transferred to infect salmon held in tanks in the Matre Research Station at the Institute of Marine Research within the same day. Maximum lice loads after infection were 2 adult females per fish. Fish were held at $12{ }^{\circ} \mathrm{C}$ under natural light conditions and fed a commercial diet daily.

To harvest egg strings from lice, fish were captured and sedated with tricaine methanesulfonate (FINQUEL, Argent Chemical Laboratories) at a concentration of $10 \mathrm{mg} \mathrm{l}^{-1}$. Lice were temporarily removed from the fish while egg strings were harvested. Up to 20 egg strings were harvested to achieve satisfactory numbers of larvae. The egg strings were transferred to an incubator system as described by Hamre et al. (2009). The incubator, date of collection and hatching date were recorded to determine larval age, and lifecycle stage was confirmed with microscopy.

\subsection{Experimental design and procedures}

To test how salinity affects larval behaviour in response to a vertical step salinity gradient, we designed a column experiment to simulate step salinity gradients and measured where the larvae preferred to distribute. We simulated haloclines with an overlying brackish layer ranging from 16-34.7 ppt on top of a fully saline layer (34.7 ppt) and tested the preferences of both nauplii and copepodid stages.

The experimental column system was comprised of 2 glass tubes $85 \mathrm{~cm}$ long and $6 \mathrm{~cm}$ in diameter. These were positioned in an outer tank which acted as a water bath to maintain a stable temperature of $12^{\circ} \mathrm{C}$ (Fig. 1). White plastic half-pipes were fitted behind the glass columns to provide sufficient visual contrast to detect larvae. Columns were continuously lit with a single $35 \mathrm{~W}$ white fluorescent light (as suggested by Aarseth \& Schram 1999), which was placed $40 \mathrm{~cm}$ above the top of the columns. This lighting produced a light intensity of $8 \mu \mathrm{mol}$ quanta $\mathrm{m}^{-2} \mathrm{~s}^{-1}$ at the water surface, similar to that described in Heuch (1995), and indicative of light levels at dawn or dusk. The
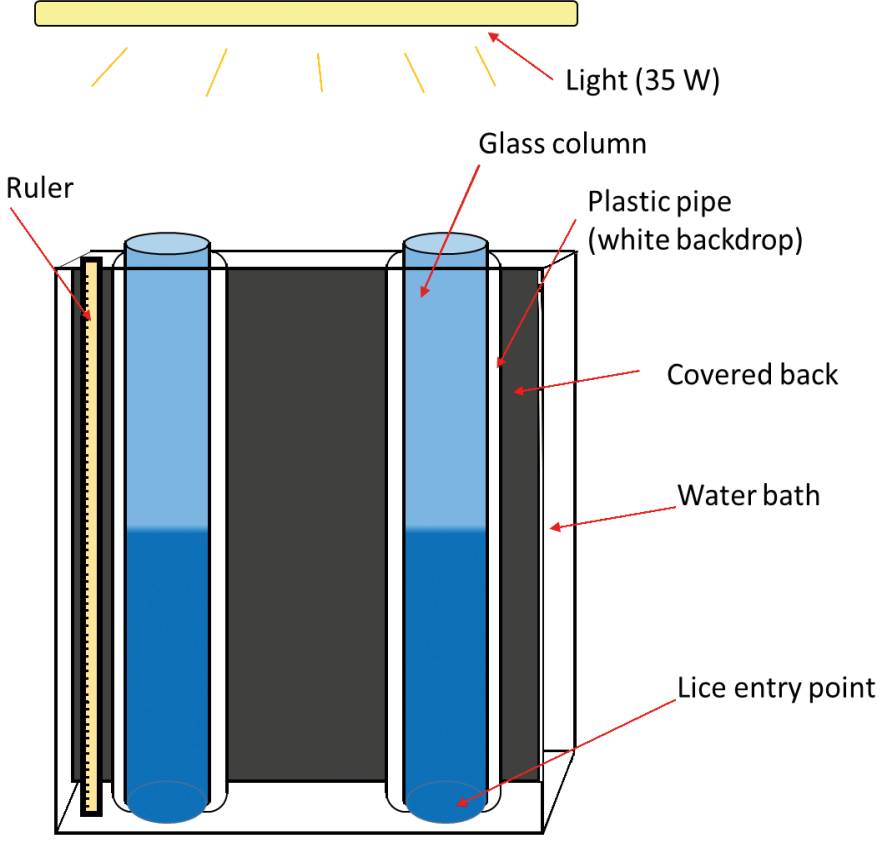

Fig. 1. Experimental column used to test the vertical distribution of nauplii and copepodid larvae of salmon lice in salinity gradients

water bath tank in which columns sat was encased in a black covering to shield columns from any ambient light. Experiments were conducted in a temperaturecontrolled room set to $12^{\circ} \mathrm{C}$.

We created a series of different haloclines at the mid-point of the column. The bottom layer was fullsalinity seawater (34.7 ppt) and the top layer varied with the following treatments: $16,18,20,22,24,26$, 28, 30, 32, and 34.7 ppt. For each halocline, we conducted 4 separate replicated column runs. The order of replicate runs was randomised across treatments.

Water of different salinities used in the treatments was created by combining filtered (rotating $40 \mu \mathrm{m}$ filter followed by UV treatment) and aerated freshwater (from local lakes and rivers) and seawater (from $95 \mathrm{~m}$ depth in nearby Masfjorden) from header tanks. The water was temperature-controlled to $12^{\circ} \mathrm{C}$. After stirring thoroughly, we used a Cond $315 \mathrm{i}$ handheld meter to confirm the mixture was within $0.1 \mathrm{ppt}$ of the desired salinity. The treatment mixture was first poured into the column up to approximately halfway $(\sim 40 \mathrm{~cm})$. Afterwards a tube was lowered to the bottom and natural saltwater (34.7 ppt) was slowly pumped in via a peristaltic pump. This resulted in a brackish overlying layer separated from the full-salinity layer by a halocline that was typically 5-10 cm thick. In all columns, a $0.5 \mathrm{~cm}$ layer of fresh water was pipetted onto the top to stop larvae from 
floating at the surface or crawling out of the water at the sides of the column.

After a halocline was established, approximately 100 lice of the appropriate stage were pipetted out of the incubators and then pumped (along with a small portion of full-salinity water) into the bottom of the column via the peristaltic pump.

\subsection{Recording salinity preference of larvae}

After lice were introduced to the column, they were given $1 \mathrm{~h}$ to distribute vertically before they were counted and their positions in the column noted on a strip of paper attached next to the tank. At count commencement, the encasing cover was removed, and a more powerful fluorescent lamp fixed right above the columns to increase lice visibility. We conducted a timed count over 2 min to mark their positions to the nearest $\mathrm{cm}$. This rapid count limited how far lice could move in response to the new light source, and so we consider the count representative of the proportions of lice throughout the column. After recording, we measured the salinity and temperature every $5 \mathrm{~cm}$ in the column with a Cond 315i handheld meter to establish how deep the halocline was located and its thickness.

\subsection{Statistical analysis}

To test if the proportion of nauplii and copepodids that migrated through the halocline to the top layer differed among treatments, 1-way ANOVAs were performed between the number of lice in the top layer of the halocline in control and treatment experiments. Tukey's tests were performed as post hoc analysis. Proportional data were logit-transformed prior to analyses and assumptions of the model were tested with diagnostic plots.

We conducted a separate analysis to assess the proportion of lice that aggregated in the halocline band within the column. To do this, we identified the $10 \mathrm{~cm}$ section of the column within which the greatest salinity change occurred. We chose a comparative halocline position in the control treatment (with $34.7 \mathrm{ppt}$ water throughout the column) by using the average location of the halocline across all other treatment groups. To test if there were differences in the proportion of lice that aggregated in the halocline among treatments, we used a 1-way ANOVA on logit-transformed proportional data followed by a post-hoc Tukey's test.

\subsection{Modelling larval dispersal}

The Norwegian NSLDM is a particle-tracking biological-physical dispersal model that currently parameterises salinity avoidance of planktonic lice larvae when salinity is <20 (Johnsen et al. 2014). To test how dispersal of lice differed when lice responses to salinity were parameterised with results from our column experiments, we ran a simulation of larvae dispersal in a fjord.

In the simulation, 100 particles, representing salmon lice during their planktonic stages, were released every hour from 2 sites (an inner and an outer position within the fjord) around the Hardangerfjord $\left(60^{\circ} \mathrm{N}, 5.5^{\circ} \mathrm{E}\right)$ on the west coast of Norway. The simulation was run for 1 mo during spring, when the fresh water input into the fjord is at its maximum (May 2016). Environmental conditions or forcing parameters for the dispersal model (water currents, temperature, and salinity) were provided by a hydrodynamic fjord model (Albretsen et al. 2011). The fjord model was set up for the study area to a horizontal resolution of $160 \times 160 \mathrm{~m}$, having 35 sigma layers in the vertical.

Lice in the model were given temperaturedependent development and the ability to migrate vertically in the water column in response to light and salinity. In previous model simulations (Johnsen et al. 2014, 2016, Samsing et al. 2016a, 2017), lice particles were given a constant upwards vertical swimming velocity $\left(0.5 \mathrm{~mm} \mathrm{~s}^{-1}\right)$ and salinity avoidance by vertical swimming when water was $20 \mathrm{ppt}$ (original model). The results from the column experiments showed that salinity avoidance increases with decreasing salinity for both the nauplii and copepodid stages. Results also showed copepodids had a stronger preference for residing close to the surface than compared to the nauplii. We implemented this in the simulation by separating between the nauplii and copepodid stages. For the copepodids, the modelled parameterised vertical swimming velocity $(w)$ was a function of salinity $(s)$. Maximum upwards swimming velocity $\left(V_{\mathrm{m}}\right)$ was set to $0.5 \mathrm{~mm} \mathrm{~s}^{-1}$, which is approximately 1 body length $\mathrm{s}^{-1}$ and should be within the speed lice can maintain over time, and maximum sinking velocity was set to $1 \mathrm{~mm} \mathrm{~s}^{-1}$ (Bricknell et al. 2006). Swimming speed as a function of the ambient salinity was therefore given by:

$$
w(s)=\left(5 \times V_{\mathrm{m}} \times \mathrm{e}^{s / 10}\right) /\left(\mathrm{e}^{34.7 / 10}\right)-4 \times V_{\mathrm{m}}
$$

For the naupliar stages, swimming towards the surface $\left(v_{\mathrm{m}}\right)$ was set to 0 . The vertical swimming directed downwards during low-salinity exposure 
was kept equal to the behaviour for the copepodids in correspondence with Eq. (1).

In addition, particles during both nauplii and copepodid stages were moved vertically in the water column by a random walk function to represent vertical mixing, given by:

$$
w(r)=r \sqrt{2\left(\frac{D_{\mathrm{v}}}{d t}\right)}
$$

Here, $r$ is a random number between -1 and $1, D_{\mathrm{v}}$ is the vertical mixing coefficient set to $10^{-3} \mathrm{~m}^{2} \mathrm{~s}^{-1}$ and $d t$ is the timestep of $180 \mathrm{~s}$. The particles during all stages were limited to the upper $15 \mathrm{~m}$ of the water column using a reflective border at the lower boundary as well as the surface. While it is possible copepodids and nauplii may occur deeper, the weight of evidence at present suggests that the majority are contained in surface waters.

In all simulations, modelled lice particles became infective copepodids at 40 degree-days and had a total lifespan of 170 degree-days (Samsing et al. 2016a). Mortality was parameterised at a constant rate of $17 \% \mathrm{~d}^{-1}$. The age and position of lice particles were stored every hour and used to calculate the density field of infective lice particles (i.e. copepodid particles).

\section{RESULTS}

\subsection{Migration through the halocline}

The nauplii stage displayed a preference for higher salinity (Fig. 2a). This was reflected in the $34.7 \mathrm{ppt}$ treatment having a higher percentage of lice in the top layer compared to any other treatment $(p<0.0001)$. Post hoc analysis showed that there was no difference between other treatments from 32 to $16 \mathrm{ppt}$.

There was a positive relationship between the proportion of copepodids in the top layer and its salinity ( $p<0.0001$; Fig. 2b). However, the relationship was more gradual than for nauplii. The percentage of lice in the top layer of the 16-30 ppt treatments differed to the control $(p<0.05)$. In all cases, a higher percentage of copepodids migrated to the top layer than nauplii undergoing the same treatment.

\subsection{Aggregation at the halocline}

Nauplii strongly aggregated in the halocline whenever it was present (Fig. 3a; $p=0.001$ ). When no halocline existed (34.7 ppt on top and bottom), nauplii were more spread throughout the column. When the overlying layer caused a step salinity gradient (16-32 ppt on top), between 31 and $44 \%$ of nauplii concentrated into the $10 \mathrm{~cm}$ wide band in the column where the halocline occurred, whereas we would only have expected about $12.5 \%$ if there had been even distribution over each $10 \mathrm{~cm}$ of the $80 \mathrm{~cm}$ column.

Copepodids were more spread in their vertical distribution in the column (Fig. 3b) but still demonstrated significant clustering at or just below the halocline when the top layer was 16 ppt ( $p=0.005)$ or $20 \mathrm{ppt}(\mathrm{p}=0.004)$ and showed a pattern for all other treatments. When no halocline existed, lice were more evenly spread throughout the column, with a small peak towards the surface. Aggregation became stronger as the overlying salinity decreased. For example, in the 16-28 ppt experiments, between 20 and $30 \%$ of copepodids concentrated into the $10 \mathrm{~cm}$ wide band in the column where the halocline occurred.

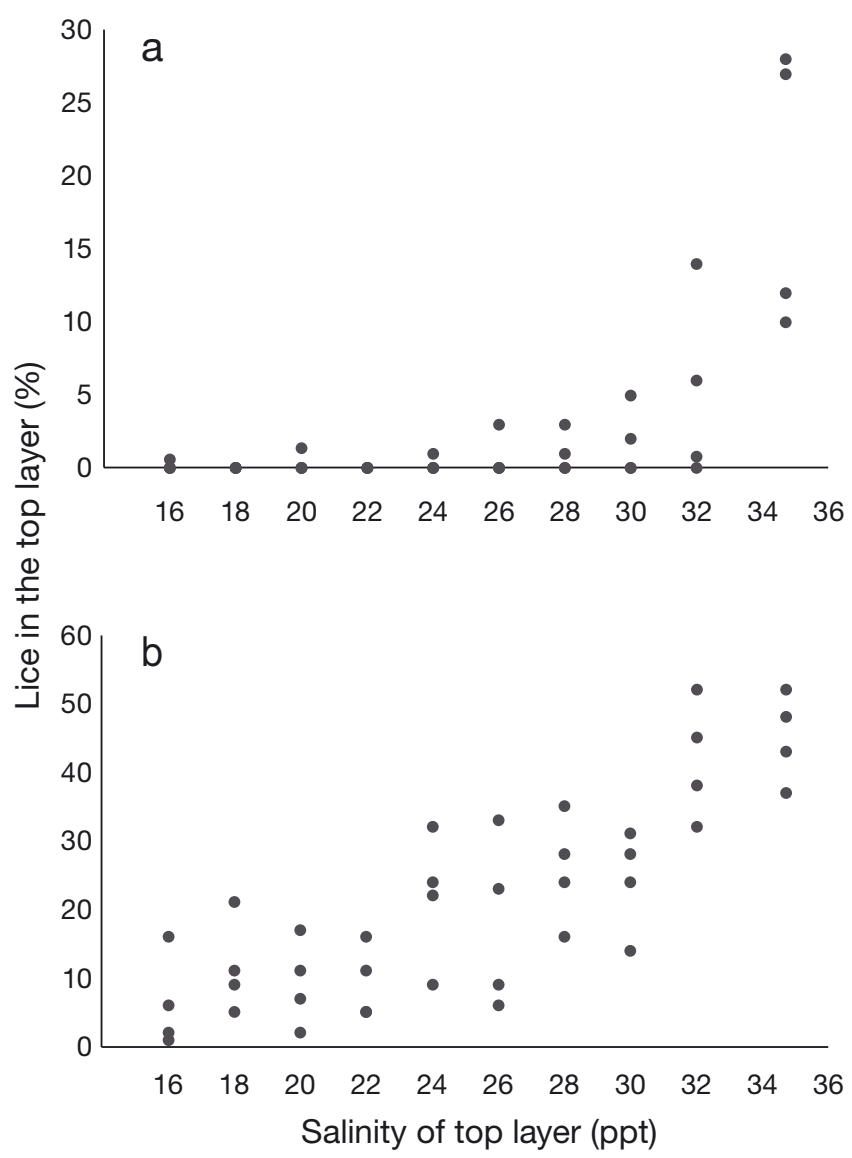

Fig. 2. Percentage of salmon lice (a) nauplii and (b) copepodids in the layer above the step salinity gradient as a function of the salinity in the overlying layer. Each point represents the proportion of lice in the top layer for a column replicate. The 34.7 ppt treatment served as an isohaline control (34.7 ppt in both the top and bottom layers of the column) 


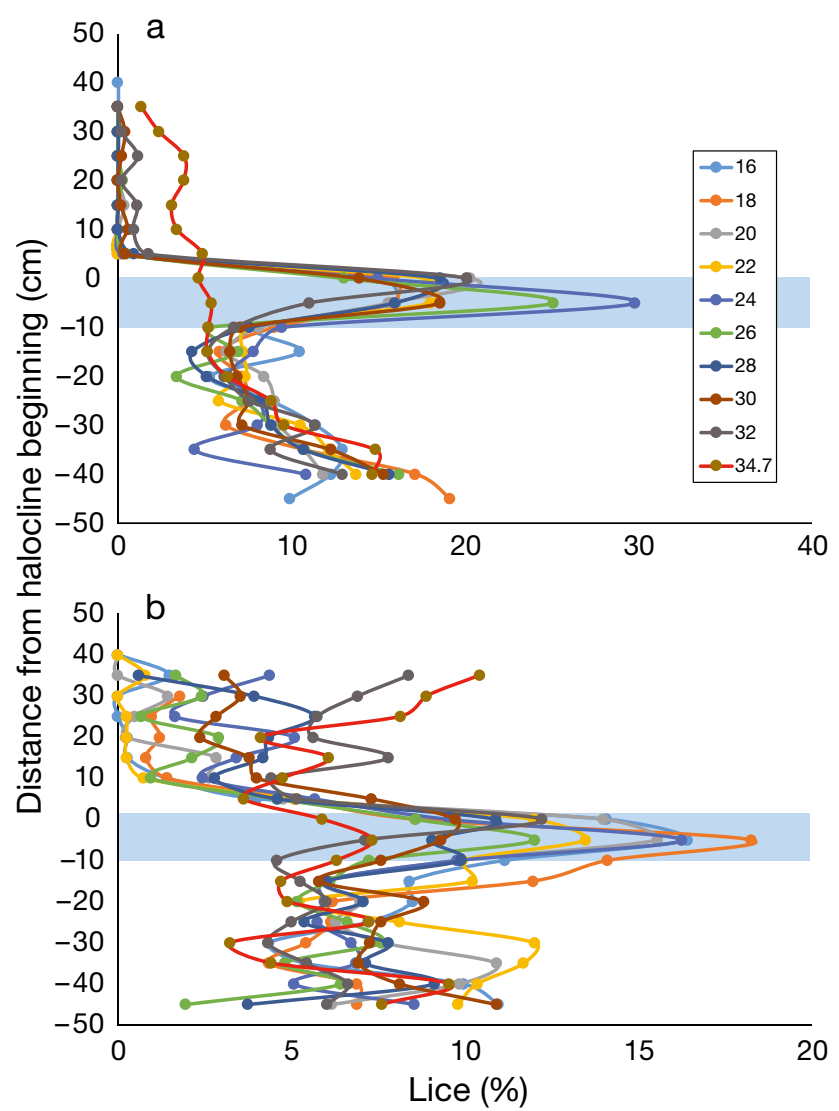

Fig. 3. Average proportion of (a) nauplii and (b) copepodids of salmon lice found in each $5 \mathrm{~cm}$ region of the column relative to the step salinity gradient. Colour key shows salinity (ppt) in the top layer for the 10 different treatments. Shaded area $=10 \mathrm{~cm}$ over which the largest change in salinity occurred. Each point represents the mean of 4 column replicates. The $34.7 \mathrm{ppt}$ treatment served as an isohaline control (34.7 ppt in both the top and bottom layers of the column)

\subsection{Modelling lice dispersal patterns}

With our 3-dimensional dispersal model, we compared vertical distributions of lice particles in the upper layers of the water column after setting a halocline at $3 \mathrm{~m}$ deep and changing the salinity above this halocline. In this experiment, simulated particles from the original model are generally distributed higher in the water column in lower salinities compared to particles in the new model simulation (Table 1). Distributions of modelled nauplii and copepodids under different salinity step gradients are given in Figs. S1 \& S2 in the Supplement at www.intres.com/articles/suppl/q011p181_supp.pdf.

The distribution of lice copepodids differed with both release location and vertical swimming behaviour in response to salinity gradients in the water column (Fig. 4). Particles released from the outer-fjord location had a mean transport distance of $\sim 4.7 \mathrm{~km}$
Table 1. Percentage of salmon lice distributed above a $3 \mathrm{~m}$ deep halocline set at different salinities using the original and the new model simulation parameters. The original model simulation sets salinity avoidance of lice particles at $20 \mathrm{ppt}$, and these have a constant swimming velocity. In the new model simulation, swimming velocity of lice particles was modelled as a function of salinity

\begin{tabular}{|c|c|c|}
\hline \multirow{2}{*}{$\begin{array}{l}\text { Salinity (ppt) } \\
\text { above halocline }\end{array}$} & \multicolumn{2}{|c|}{ Distribution (\%) of lice } \\
\hline & Original model & New model \\
\hline 16 & 33.3 & 13 \\
\hline 18 & 33.9 & 14 \\
\hline 20 & 78.9 & 16 \\
\hline 22 & 78.2 & 18 \\
\hline 24 & 79.0 & 20 \\
\hline 26 & 79.5 & 25 \\
\hline 28 & 79.4 & 31 \\
\hline 30 & 79.2 & 40 \\
\hline 32 & 79.2 & 57 \\
\hline 34 & 79.2 & 77 \\
\hline 34.7 & 82.0 & 87 \\
\hline
\end{tabular}

when salinity avoidance was parameterised at $20 \mathrm{ppt}$ (original model; Fig. 4a) and a larger dispersal when salinity was parameterised with findings from the present column experiment ( $6.9 \mathrm{~km}$ in the new model) (Fig. 4c). In contrast, when lice particles were released from an inner-fjord location, mean transport distance was almost 3 times longer when salinity avoidance was parameterised at 20 ppt ( 28.9 km; Fig. 4b) compared to when it was parameterised with findings from the column experiment ( 11.0 km; Fig. 4d).

\section{DISCUSSION}

We have demonstrated that both nauplii and copepodids have distinct vertical distributions in response to salinity step gradients and exhibit strong aggregative behaviour towards the halocline layer. Nauplii display greater preference for high seawater salinity compared to brackish water and display greater avoidance earlier, completely avoiding the top layer at salinities where copepodids could still be found. As the responses of nauplii towards salinity have previously been considered analogous to copepodids and modelled in the same way (e.g. Johnsen et al. 2014), our results represent an important advance. Our findings have implications for understanding the distribution and dispersal of salmon lice in coastal waters.

\subsection{Vertical distribution of nauplii and copepodids}

In step salinity experiments, nauplii responded strongly to avoid brackish water. As they are the ear- 

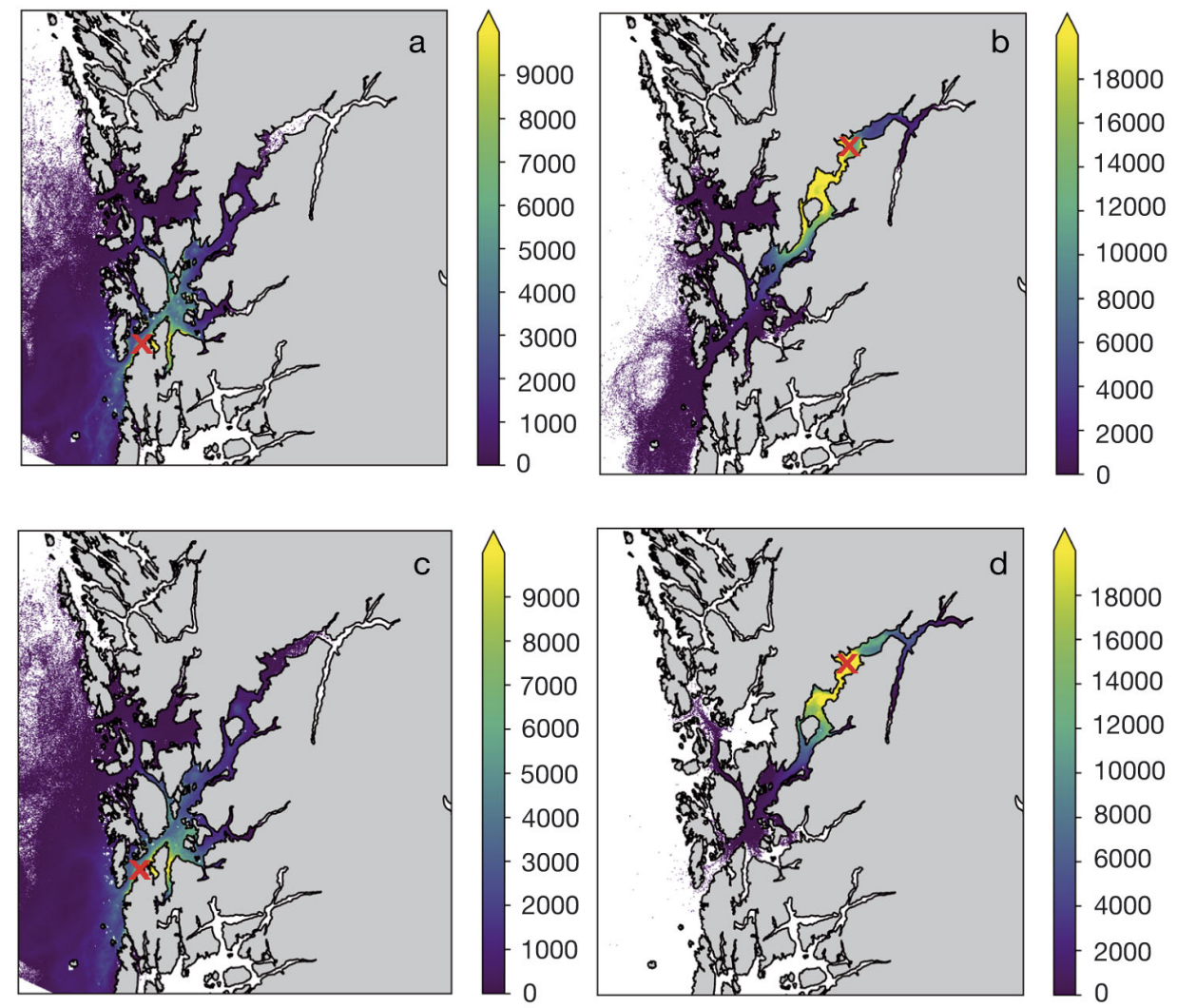

Fig. 4. Salmon lice particle density per model grid $(160 \times 160 \mathrm{~m})$ on the southwest coast of Norway during May 2016 . Red cross marks the position of particle release. Copepodid distribution after release from (a) a coastal site or (b) an inner fjord site using the existing dispersal model parameterisation of salinity avoidance by lice larvae at 20 ppt; (c) a coastal site or (d) an inner fjord site using the new dispersal model parameterisation with results from the present study

liest and smallest larval forms, nauplii have the least tolerance for low salinities (Gravil 1996). They must develop into copepodids before they can infect a host and are therefore less likely to respond to cues that could assist in host finding. For instance, they are less sensitive to light (Novales Flamarique et al. 2000) and they show weaker diel migration than copepodids (Heuch et al. 1995). Nauplii in isohaline columns did not distribute evenly, as did the copepodids, which reflects their reduced responsiveness to light via less upwards swimming. Our results may indicate that nauplii have a deeper distribution than copepodids under many environmental settings. Groundtruthing of the nauplii results in larger columns and natural settings is required. Further, the influence of temperature gradients on vertical distribution is unknown and should be tested.

Copepodids demonstrated aversive behaviours to lower salinities, but avoidance was less extreme. They were present in similar percentages when the upper layer was 32 and $34.7 \mathrm{ppt}$, with lower percentages present as salinity decreased. Even at very brackish salinities of 16-20 ppt, some copepodids $(\sim 10 \%)$ were found above the halocline. This indicates more tolerance for brackish water than nauplii in general, even if their preference is for high salinity (Heuch 1995, Gravil 1996). As lecithotrophic larvae, copepodids must detect and attach to a host before their energy reserves deplete. Their wild hosts, Atlantic salmon smolts out-migrating from rivers and sea trout Salmo trutta, mostly swim in the upper 1-3 m of water (Davidsen et al. 2009, Thorstad et al. 2012, Eldøy et al. 2017). Further, farmed salmon spend much of their time in surface waters (Oppedal et al. 2011). To maximise their encounter possibility with a host, copepodids respond strongly to light cues (Novales Flamarique et al. 2000) and swim upwards into surface waters until low salinities are encountered.

The accumulation in the halocline we observed may thus be due to an equilibrium between attraction to surface light and avoidance of the brackish top layer. This would explain why aggregation of less light-sensitive nauplii in the halocline was stronger than for copepodids. However, the phenomenon was still present when a proportion of the copepodids tolerated the salinity of the water layer above. Attrac- 
tion to a step salinity gradient is a behaviour documented for many zooplankton (Harder 1968), and in the case of the salmon louse, likely improves host finding. Copepodids can detect waterborne semiochemicals originating from salmon (Devine et al. 2000, Bailey et al. 2006) and would get optimum use of this sense at a step gradient where olfactory cues are better preserved than in a homogeneous water column (Steele 1978, Westerberg 1989).

During out-migration, salmon smolts transform from a freshwater fish to becoming salt-tolerant (Cunjak et al. 1990, Davidsen et al. 2009, Thorstad et al. 2012). Migration mainly occurs in the top $3 \mathrm{~m}$ of water at an average swimming depth of $1.7 \mathrm{~m}$ (Plantalech Manella et al. 2009). Likewise, the salmon louse's other major host species, the sea trout, spends $90 \%$ of its time in the top $3 \mathrm{~m}$ of water (Klemetsen et al. 2003, Rikardsen et al. 2007). For these species, frequent crosses of isohalines are seen and vertical movement may be performed to help avoid predators and search for prey (Plantalech Manella et al. 2009). It is not unreasonable that they would spend time in step salinity gradients to feed on aggregating plankton (Harder 1968), and so lice have evolved behaviours to seek out these step gradients as well.

\subsection{Implications of larval salinity preferences for predicting lice vertical distribution and dispersal}

Depth-based technologies used in salmon farms to prevent lice infection work on the principle of shielding or re-distributing salmon from where lice most commonly aggregate. These include physical barriers such as snorkel cages (Stien et al. 2016, Oppedal et al. 2017, Wright et al. 2017), skirts (Grøntvedt et al. 2018, Stien et al. 2018), as well as deep lights and feeding (Frenzl et al. 2014, Stien et al. 2014), which aim to keep salmon below where the lice are present. Lice are presumed to concentrate at the surface, with strong evidence for this pattern in unstratified conditions (Murray \& Gillibrand 2006, Samsing et al. 2016a, Stien et al. 2016) and a clear reduction in infestation pressure as salmon are held deeper (Oppedal et al. 2017). These technologies are used in a static way, protecting the same cage depths for large blocks of time after deployment. This may prove adequate when the water column is unstratified, but our results demonstrating the importance of the halocline for lice aggregation indicate that a more dynamic strategy will be more effective in preventing infection when stratification is present (Samsing et al. 2016a).
The finer-scale knowledge of copepodid gradient preferences we have reported enables implementation of depth-dynamic preventative methods. For instance, farmers can manipulate the depth of a snorkel or skirt (Oppedal et al. 2017), or alter the depths of feeding points and lights so that it best protects the cage. In situations with a fresher surface layer and a deep halocline, it may even be important to encourage fish upwards away from aggregations of copepodids that would form at the deeper halocline.

When applied to modelling salmon louse dispersal, our results changed the expected horizontal distribution of larvae. This difference in the transport distance is due to changes of lice vertical distribution in the water column (Asplin et al. 2014, Johnsen et al. 2014). Models parameterised with a sharp 20 ppt cut off for salinity avoidance for both nauplii and copepodids simulated a considerably higher proportion of lice in brackish water above $20 \mathrm{ppt}$, which led to greater dispersal by the vertically stratified currents (Johnsen et al. 2014). The new salinity parameters predict much stronger avoidance of layers below full salinity and only allow for upward swimming when salinity is above $\sim 32.5$ ppt. As a result, a much larger proportion of nauplii and to a lesser degree copepodids are simulated as deeper in the water column. The contrast between the old and new parameters means that transport distance differs most when strong stratification exists. Spatially, this difference is more pronounced in inner fjord locations compared to coastal locations, where vertical mixing is more prevalent. In addition to its influence on vertical distribution, salinity will influence daily mortality rates (Johnson \& Albright 1991, Bricknell et al. 2006), with consequences for the number of infection-competent copepods that are dispersed; further adjustments to dispersal models are required to fully capture this.

As our experiment used $1 \mathrm{~m}$ high columns, pressure was a negligible factor. Lice larvae might swim upward in response to pressure, a response which could interact with salinity, light and other preferences to determine vertical distribution. The depths copepodids are simulated to sink to in the present model are deeper than earlier observed (Penston et al. 2008); however, these observations were not made deeper than $5 \mathrm{~m}$, nor was the stratification as strong as the case for Norwegian fjords during peak runoff. In situ observations of planktonic lice vertical distribution under highly stratified conditions would be valuable to evaluate the discrepancy between model and observed distributions. Further experiments conducted in large or pressurised columns will also help define the possible influence of pressure on larval 
swimming responses and better set realistic depth parameters for dispersal modelling. Ground-truthing of these results in large columns and in the field is required to ensure that they are representative of lice vertical distributions in natural settings, where light conditions, turbulence, turbidity and other influences related to the scale of experimentation in small columns may differ substantially.

Acknowledgements. We thank Sussie Dalvin, Samantha Bui and Rasmus Skern-Mauritzen (Institute of Marine Research) for discussions regarding the vertical distribution of sea lice larvae. We also thank staff at the Institute of Marine Research's Matre Research Station for technical assistance during the experiment, including Ivar Helge Matre, Tone Vågseth and Simon Flavell.

\section{LITERATURE CITED}

Aarseth KA, Schram TA (1999) Wavelength-specific behaviour in Lepeophtheirus salmonis and Calanus finmarchicus to utraviolet and visible light in laboratory experiments (Crustacea: Copepoda). Mar Ecol Prog Ser 186: 211-217

Abolofia J, Asche F, Wilen JE (2017) The cost of lice: quantifying the impacts of parasitic sea lice on farmed salmon. Mar Resour Econ 32:329-349

Allen SE, Lewis AG (2013) Lepeophtheirus salmonis (Krøyer, 1837): second nauplius and copepodid locomotor appendages, surface areas and possible appendage functions. Crustaceana 86:1695-1710

Norði GA, Simonsen K, Danielsen E, Eliasen K and others (2015) Abundance and distribution of planktonic Lepeophtheirus salmonis and Caligus elongatus in a fish farming region in the Faroe Islands. Aquacult Environ Interact $7: 15-27$

Asplin L (2011) NorKyst-800 report no. 1: user manual and technical descriptions. IMR Res Rep Ser Fisken og Havet 2/2011. Institute of Marine Research, Bergen

Asplin L, Johnsen IA, Sandvik AD, Albretsen J, Sundfjord V, Aure J, Boxaspen KK (2014) Dispersion of salmon lice in the Hardangerfjord. Mar Biol Res 10:216-225

*Bailey RJ, Birkett MA, Ingvarsdóttir A, Mordue Luntz AJ and others (2006) The role of semiochemicals in host location and non-host avoidance by salmon louse (Lepeophtheirus salmonis) copepodids. Can J Fish Aquat Sci 63:448-456

Bjørn PA, Finstad B, Kristoffersen R (2001) Salmon lice infection of wild sea trout and Arctic char in marine and freshwaters: the effects of salmon farms. Aquacult Res 32:947-962

Bricknell IR, Dalesman SJ, O'Shea B, Pert CC, Luntz AJ (2006) Effect of environmental salinity on sea lice Lepeophtheirus salmonis settlement success. Dis Aquat Org 71:201-212

* Bui S, Dempster T, Remen M, Oppedal F (2016) Effect of ectoparasite infestation density and life-history stages on the swimming performance of Atlantic salmon Salmo salar. Aquacult Environ Interact 8:387-395

Cunjak RA, Saunders RL, Chadwick EMP (1990) Seasonal variations in the smolt characteristics of juvenile Atlantic salmon (Salmo salar) from estuarine and riverine environments. Can J Fish Aquat Sci 47:813-820
Davidsen JG, Rikardsen AH, Halttunen E, Thorstad EB and others (2009) Migratory behaviour and survival rates of wild northern Atlantic salmon Salmo salar post-smolts: effects of environmental factors. J Fish Biol 75:1700-1718

*Dawson LHJ, Pike AW, Houlihan DF, McVicar AH (1999) Changes in physiological parameters and feeding behaviour of Atlantic salmon Salmo salar infected with sea lice Lepeophtheirus salmonis. Dis Aquat Organ 35:89-99

* Devine GJ, Ingvarsdóttir A, Mordue W, Pike AW, Pickett J, Duce I, Mordue AJ (2000) Salmon lice, Lepeophtheirus salmonis, exhibit specific chemotactic responses to semiochemicals originating from the salmonid, Salmo salar. J Chem Ecol 26:1833-1847

Eldøy SH, Davidsen JG, Thorstad EB, Whoriskey FG and others (2017) Marine depth use of sea trout Salmo trutta in fjord areas of central Norway. J Fish Biol 91:1268-1283

Frenzl B, Stien LH, Cockerill D, Oppedal F and others (2014) Manipulation of farmed Atlantic salmon swimming behaviour through the adjustment of lighting and feeding regimes as a tool for salmon lice control. Aquaculture 424-425:183-188

Gravil HR (1996) Studies on the biology and ecology of the free swimming larval stages of Lepeophtheirus salmonis (Kroyer, 1838) and Caligus elongatus Nordmann, 1832 (Copepoda: Caligidae). MSc thesis, Stirling University

Grøntvedt RN, Kristoffersen AB, Jansen PA (2018) Reduced exposure of farmed salmon to salmon louse (Lepeophtheirus salmonis L.) infestation by use of plankton nets: estimating the shielding effect. Aquaculture 495:865-872

*Hamre LA, Glover KA, Nilsen F (2009) Establishment and characterisation of salmon louse (Lepeophtheirus salmonis (Krøyer 1837)) laboratory strains. Parasitol Int 58: 451-460

*Hamre LA, Eichner C, Caipang CM, Dalvin ST and others (2013) The salmon louse Lepeophtheirus salmonis (Copepoda: Caligidae) life cycle has only two chalimus stages. PLOS ONE 8:e73539

*Harder W (1968) Reactions of plankton organisms to water stratification. Limnol Oceanogr 13:156-169

* Heuch PA (1995) Experimental evidence for aggregation of salmon louse copepodids (Lepeophtheirus salmonis) in step salinity gradients. J Mar Biol Assoc UK 75:927-939

*Heuch PA, Parsons A, Boxaspen K (1995) Diel vertical migration: a possible host-finding mechanism in salmon louse (Lepeophtheirus salmonis) copepodids? Can J Fish Aquat Sci 52:681-689

Johnsen IA, Fiksen Ø, Sandvik AD, Asplin L (2014) Vertical salmon lice behaviour as a response to environmental conditions and its influence on regional dispersion in a fjord system. Aquacult Environ Interact 5:127-141

Johnsen IA, Asplin LC, Sandvik AD, Serra-Llinares RM (2016) Salmon lice dispersion in a northern Norwegian fjord system and the impact of vertical movements. Aquacult Environ Interact 8:99-116

Johnson SC, Albright LJ (1991) Development, growth, and survival of Lepeophtheirus salmonis (Copepoda: Caligidae) under laboratory conditions. J Mar Biol Assoc UK 71:425-436

Kinlan BP, Gaines SD, Lester SE (2005) Propagule dispersal and the scales of marine community process. Divers Distrib 11:139-148

Klemetsen A, Amundsen PA, Dempson JB, Jonsson B, Jonsson N, O'Connell MF, Mortensen E (2003) Atlantic salmon Salmo salar L., brown trout Salmo trutta L. and Arctic charr Salvelinus alpinus (L.): a review of aspects of their life histories. Ecol Freshw Fish 12:1-59

Kristoffersen AB, Rees EE, Stryhn H, Ibarra R, Campisto JL, 
Revie CW, St-Hilaire S (2013) Understanding sources of sea lice for salmon farms in Chile. Prev Vet Med 111: $165-175$

Kristoffersen AB, Qviller L, Helgesen KO, Vollset KW, Viljugrein H, Jansen PA (2018) Quantitative risk assessment of salmon louse-induced mortality of seaward-migrating post-smolt Atlantic salmon. Epidemics 23:19-33

Lovdata (2018) Forskrift om bekjempelse av lakselus i akvakulturanlegg [Regulations on the control of salmon lice in aquaculture farms]. Ministry of Trade, Industry and Fisheries, Oslo. https://lovdata.no/dokument/SF/ forskrift/2012-12-05-1140

Murray AG, Gillibrand PA (2006) Modelling salmon lice dispersal in Loch Torridon, Scotland. Mar Pollut Bull 53:128-135

Myksvoll MS, Sandvik AD, Albretsen J, Asplin L and others (2018) Evaluation of a national operational salmon lice monitoring system-from physics to fish. PLOS ONE 13:e0201338

Novales Flamarique I, Browman HI, Bélanger M, Boxaspen K (2000) Ontogenetic changes in visual sensitivity of the parasitic salmon louse Lepeophtheirus salmonis. J Exp Biol 203:1649-1657

\% Oppedal F, Dempster T, Stien LH (2011) Environmental drivers of Atlantic salmon behaviour in sea-cages: a review. Aquaculture 311:1-18

Oppedal F, Samsing F, Dempster T, Wright DW, Bui S, Stien LH (2017) Sea lice infestation levels decrease with deeper 'snorkel' barriers in Atlantic salmon sea-cages. Pest Manage Sci 73:1935-1943

Øverli Ø, Nordgreen J, Mejdell CM, Janczak AM, Kittilsen S, Johansen IB, Horsberg TE (2014) Ectoparasitic sea lice (Lepeophtheirus salmonis) affect behavior and brain serotonergic activity in Atlantic salmon (Salmo salar L.): perspectives on animal welfare. Physiol Behav 132:44-50

Overton K, Samsing F, Oppedal F, Dalvin S, Stien LH, Dempster T (2018a) The use and effects of hydrogen peroxide on salmon lice and post-smolt Atlantic salmon. Aquaculture 486:246-252

Overton K, Dempster T, Oppedal F, Kristiansen TS, Gismervik K, Stien LH (2018b) Salmon lice treatments and salmon mortality in Norwegian aquaculture: a review. Rev Aquacult, doi:10.1111/raq.12299

Penston MJ, Millar CP, Zuur A, Davies IM (2008) Spatial and temporal distribution of Lepeophtheirus salmonis (Krøyer) larvae in a sea loch containing Atlantic salmon, Salmo salar L., farms on the north-west coast of Scotland. J Fish Dis 31:361-371

* Plantalech Manella N, Thorstad EB, Davidsen JG, Økland F, Sivertsgård R, Mckinley RS, Finstad B (2009) Vertical movements of Atlantic salmon post-smolts relative to measures of salinity and water temperature during the first phase of the marine migration. Fish Manage Ecol 16:147-154

Rikardsen AH, Diserud OH, Elliott JM, Dempson JB, Sturlaugsson J, Jensen AJ (2007) The marine temperature and depth preferences of Arctic charr (Salvelinus alpinus) and sea trout (Salmo trutta), as recorded by data storage tags. Fish Oceanogr 16:436-447

Samsing F, Johnsen I, Stien LH, Oppedal F, Albretsen J, Asplin L, Dempster T (2016a) Predicting the effectiveness of depth-based technologies to prevent salmon lice infection using a dispersal model. Prev Vet Med 129:48-57

Samsing F, Oppedal F, Dalvin S, Johnsen I, Vågseth T,
Dempster T (2016b) Salmon lice (Lepeophtheirus salmonis) development times, body size, and reproductive outputs follow universal models of temperature dependence. Can J Fish Aquat Sci 73:1841-1851

* Samsing F, Johnsen I, Dempster T, Oppedal F, Treml EA (2017) Network analysis reveals strong seasonality in the dispersal of a marine parasite and identifies areas for coordinated management. Landsc Ecol 32:1953-1967

Steele JH (1978) Some comments on plankton patches. In: Steele JH (eds) Spatial pattern in plankton communities. NATO Conference Series (IV Marine Sciences), Vol 3. Springer, Boston, MA

Stien LH, Fosseidengen JE, Malm ME, Sveier H, Torgersen T, Wright DW, Oppedal F (2014) Low intensity light of different colours modifies Atlantic salmon depth use. Aquacult Eng 62:42-48

Stien LH, Dempster T, Bui S, Glaropoulos A, Fosseidengen JE, Wright DW, Oppedal F (2016) 'Snorkel' sea lice barrier technology reduces sea lice loads on harvest-sized Atlantic salmon with minimal welfare impacts. Aquaculture 458:29-37

* Stien LH, Lind MB, Oppedal F, Wright DW, Seternes T (2018) Skirts on salmon production cages reduced salmon lice infestations without affecting fish welfare. Aquaculture 490:281-287

Thorstad EB, Whoriskey F, Uglem I, Moore A, Rikardsen AH, Finstad B (2012) A critical life stage of the Atlantic salmon Salmo salar: behaviour and survival during the smolt and initial post-smolt migration. J Fish Biol 81: $500-542$

Torrissen O, Jones S, Asche F, Guttormsen A and others (2013) Salmon lice-impact on wild salmonids and salmon aquaculture. J Fish Dis 36:171-194

* Treml EA, Halpin PN, Urban DL, Pratson LF (2008) Modeling population connectivity by ocean currents, a graphtheoretic approach for marine conservation. Landsc Ecol 23:19-36

* Tucker CS, Sommerville C, Wootten R (2000) An investigation into the larval energetics and settlement of the sea louse, Lepeophtheirus salmonis, an ectoparasitic copepod of Atlantic salmon, Salmo salar. Fish Pathol 35: 137-143

Tully O, Nolan DT (2002) A review of the population biology and host-parasite interactions of the sea louse Lepeophtheirus salmonis (Copepoda: Caligidae). Parasitology 124:165-182

*Vollset KW, Dohoo I, Karlsen $\varnothing$, Halttunen E and others (2018) Disentangling the role of sea lice on the marine survival of Atlantic salmon. ICES J Mar Sci 75:50-60

*Wagner GN, McKinley RS (2004) Anaemia and salmonid swimming performance: the potential effects of sublethal sea lice infection. J Fish Biol 64:1027-1038

Westerberg H (1989) Properties of aquatic odour trails. In: Døving KB (ed) Proc Tenth Int Symp Olfaction and Taste, Oslo, Norway, p 45-54

W Wright DW, Oppedal F, Dempster T (2016) Early-stage sea lice recruits on Atlantic salmon are freshwater sensitive. J Fish Dis 39:1179-1186

W Wright DW, Stien LH, Dempster T, Vagseth T, Nola V, Fosseidengen JE, Oppedal F (2017) 'Snorkel' lice barrier technology reduced two co-occurring parasites, the salmon louse (Lepeophtheirus salmonis) and the amoebic gill disease causing agent (Neoparamoeba perurans), in commercial salmon sea-cages. Prev Vet Med 140:97-105

Submitted: November 15, 2018; Accepted: February 8, 2019 Proofs received from author(s): April 23, 2019 